\title{
Review: The roles and functions of glutamine on intestinal health and performance of weaning pigs
}

\author{
F. J. Ji1,2,3a, L. X. Wang ${ }^{1,3 a}$, H. S. Yang ${ }^{1,3 \dagger} \oplus$, A. Hu ${ }^{1}$ and Y. L. Yin ${ }^{1,3,4}$
}

${ }^{1}$ Hunan International Joint Laboratory of Animal Intestinal Ecology and Health, Laboratory of Animal Nutrition and Human Health, College of Life Sciences, Hunan Normal University, 36 Lushan Road, Changsha 410081, People's Republic of China; ${ }^{2}$ Tropical Crops Genetic Resources Institute, Chinese Academy of Tropical Agricultural Sciences, 4 West Xueyuan Road, Haikou 571101, People's Republic of China; ${ }^{3}$ Hunan Provincial Key Laboratory of Animal Nutritional Physiology and Metabolic Process; National Engineering Laboratory for Pollution Control and Waste Utilization in Livestock and Poultry Production, Institute of Subtropical Agriculture, Chinese Academy of Sciences, 644 Yuanda 2nd Road, Changsha 410125, People's Republic of China; ${ }^{4}$ Academician Workstation of Changsha Medical University, 1501 Leifeng Road, Changsha 410219, People's Republic of China

(Received 18 December 2018; Accepted 15 July 2019; First published online 13 August 2019)

The gut is composed of a single layer of intestinal epithelial cells and plays important roles in the digestion and absorption of nutrients, immune and barrier functions and amino acid metabolism. Weaning stress impairs piglet intestinal epithelium structural and functional integrities, which results in reduced feed intake, growth rates and increased morbidity and mortality. Several measures are needed to maintain swine gut development and growth performance after weaning stress. A large body of evidence indicates that, in weaning piglets, glutamine, a functional amino acid, may improve growth performance and intestinal morphology, reduce oxidative damage, stimulate enterocyte proliferation, modulate cell survival and death and enhance intestinal paracellular permeability. This review focuses on the effects of glutamine on intestinal health in piglets. The aim is to provide evidentiary support for using glutamine as a feed additive to alleviate weaning stress.

Keywords: swine, weaning stress, functional amino acid, barrier function, epithelial cell renewal

\section{Implications}

Piglets are subject to constant nutritional, psychological and environmental stresses during weaning, which can result in significant swine industry economic losses. Developing strategies to reduce the negative consequences of post-weaning stress is of great concern. Glutamine is an important functional amino acid. It alleviates growth suppression and repairs weaning piglet intestinal epithelium. This article summarizes recent researches which highlight the potential use of glutamine in pig nutrition and underlying mechanisms involved. This review helps to provide references for glutamine use in swine production.

\section{Introduction}

The gastrointestinal tract plays crucial roles in digestion and absorption of nutrients, providing barrier functions, as well as amino acid metabolism (Wijtten et al., 2011). Its health is vitally important to host growth and development. With the widespread application of early weaning, piglets are

aThese two authors contributed equally to this work.

†E-mail: yhs@hunnu.edu.cn immediately exposed to many environmental and psychosocial stressors that impact on gut structure and function (Spreeuwenberg et al., 2001; Yin et al., 2014). These changes cause abnormal digestion and absorption, poor growth performance and increased diarrhea rates (Montagne et al., 2007). Exploring different nutritional factors, management and strategies that focus on promoting weaning pig growth are critical to optimizing performance. Glutamine has always been classified as a nonessential amino acid when assigning swine nutritional requirement. One of the major reasons for this is that glutamine naturally occurs in conventional feed ingredients such as corn grain, sorghum grain, soybean meal and meat and bone meal. Natural glutamine constitutes about $10 \%$ of the amino acid content of total dietary protein $(\mathrm{Wu}, 2014)$. Also, post-weaning pigs weighing around 5 to $10 \mathrm{~kg}$ have high net glutamine synthetic rates (up to 1149 $\mathrm{mg} / \mathrm{kg}$ BW per day) in their extraintestinal tissues ( $\mathrm{Wu}$, 2014). However, all tissues utilize glutamine (Wu et al., 2010), and there is growing evidence that pigs have a dietary requirement for glutamine to achieve maximum growth performance and facilitate normal intestinal physiology, particularly in hypercatabolic states (Wang et al., 2008; Blachier et al., 2013; Wu, 2014). As swine nutrition practices have 
Ji, Wang, Yang, Hu and Yin

Table 1 The common effects of weaning on piglet performance and intestinal development and function

\begin{tabular}{|c|c|c|c|}
\hline Piglets & Weaning phase & Significant result & References \\
\hline 14-day weaning & Days $1,3,5,7$ & $\begin{array}{l}\downarrow \text { body weight; } \downarrow \text { diamine oxidase and D-lactate concentrations } \\
\downarrow \text { PCNA-positive cells; } \downarrow \text { AKP activity; } \downarrow \text { E-cadherin, occludin and } \\
\text { zonula occludens (ZO)-1 mRNA or protein abundances }\end{array}$ & Wang et al. (2016b) \\
\hline 19-day weaning & Days $1,2,7$ & $\begin{array}{l}\downarrow \text { organ weight; } \downarrow \text { small intestine length } \\
\downarrow \text { lactase, maltase, dipeptidyl-peptidase } 4 \text {; amino-peptidase } \mathrm{N} \text { activities } \\
\uparrow \text { transepithelial electrical resistance; } \\
\uparrow[3 \mathrm{H}] \text { mannitol; } \uparrow \text { serum CRF }\end{array}$ & Montagne et al. (2007) \\
\hline 21-day weaning & Days $0.5,1,2,4,7$ & $\begin{array}{l}\downarrow \text { average cumulative intake; } \\
\downarrow \text { goblet cells numbers } \\
\uparrow \text { T lymphocytes numbers; } \\
\uparrow \text { metalloproteinase stromelysin expression } \\
\downarrow \text { jejunal villus height; } \uparrow \text { crypt depth; } \\
\downarrow \text { MHC class I RNA expression }\end{array}$ & Mccracken et al. (1999) \\
\hline 21-day weaning & Days $0,2,5,8,15$ & $\begin{array}{l}\downarrow \text { relative weight of the small intestine; } \\
\uparrow \text { increases in intestinal permeability } \\
\downarrow \text { pancreatic parameters; } \downarrow \text { villus height }\end{array}$ & Moeser et al. (2007) \\
\hline 21-day weaning & Days $0,2,5,8,15$ & $\begin{array}{l}\downarrow \text { villous height; } \uparrow \text { crypt length; } \\
\downarrow \text { lactase activity } \\
\downarrow 5 \text {-HT, theophylline and HRP fluxes }\end{array}$ & Boudry et al. (2004) \\
\hline
\end{tabular}

PCNA = proliferating cell nuclear antigen; $A K P=$ alkaline phosphatase; $C R F=$ corticotropin-releasing factor; $M H C=$ major histocompatibility complex 5 - $\mathrm{HT}=5$-hydroxytryptamine; $\mathrm{HRP}=$ horseradish peroxidase.

rapidly changed, the understanding of the underlying mechanisms needs to keep pace. This article provides an overview on the effects of weaning on gastrointestinal structure and function, the effects of glutamine on piglet performance and gut health and information on the underlying mechanisms, and how they are influenced by glutamine.

\section{Intestinal development and health after weaning}

A multitude of factors contribute to post-weaning stress, including nutritional (change from sow's milk to solid feed), psychological (mixing of different litters and removal from the sow), environmental (moving from farrowing crates to nursery pens) and others (Moeser et al., 2007; Yin et al., 2014). Weaning stress is generally accompanied by reduced feed intake, decreased nutrient utilization, poor growth performance and increased disease susceptibility (Montagne et al., 2007). Several articles demonstrate that weaning stress also negatively impacts intestinal development and function (Montagne et al., 2007). This review highlights the effects of weaning stress on intestinal epithelial development and its redox status. The common effects of weaning on piglet performance and intestinal development are provided in Table 1.

Intestinal epithelium developmental changes after weaning The gut includes crypts and villi, which are formed by four principal cell types: absorptive enterocytes, mucus-producing goblet cells, hormone-secreting enteroendocrine cells and lysozyme-containing Paneth cells (Chen et al., 2018a). The porcine intestinal epithelium structure is shown in Figure 1. The homeostasis and renewal of the intestinal epithelium involve stem cell proliferation at the base of the crypt bottom, cell population expansion in the middle of the villus, terminal differentiation in the upper villus and senescent cell extrusion at the villus tip (Yang et al., 2016b). This intricate process of epithelial cells along the crypt-villus axis is accompanied by functional specialization in the small intestine (Yang et al., 2016a). The proliferation, differentiation and apoptosis of intestinal epithelial cells play important roles in intestinal development, maintenance and recovery from stress-induced tissue damage. In weaning piglets, the altered balance between crypt cell proliferation and epithelial cell apoptosis causes intestinal villus atrophy and crypt hyperplasia resulting in a loss in nutrient absorptive surfaces (Montagne et al., 2007). There is a decrease in enterocyte differentiation post-weaning accompanied by the loss of digestive enzymes, such as lactase and sucrase, and thus a decrease in intestinal digestive capacity (Pluske et al., 1997).

\section{Post-weaning distinctive intestinal functional characteristics}

An increase in reactive oxygen species (ROS) can induce oxidative stress during animal growth and development, and weaning is often associated with oxidative stress and decreased antioxidant capacity in the intestine and other tissues of pigs (Wang et al., 2008; Yin et al., 2014). For example, weaning pigs at 14 days of age resulted in oxidative injury to lipid, protein and DNA and increased the plasma concentrations of damage biomarkers such as malondialdehyde, protein carbonyl and 8-hydroxydeoxyguanosine (Yin et al., 2014). Concurrently, the activity of many antioxidant enzymes, such as superoxide dismutase, catalase and glutathione peroxidase, is also reduced due to weaning 


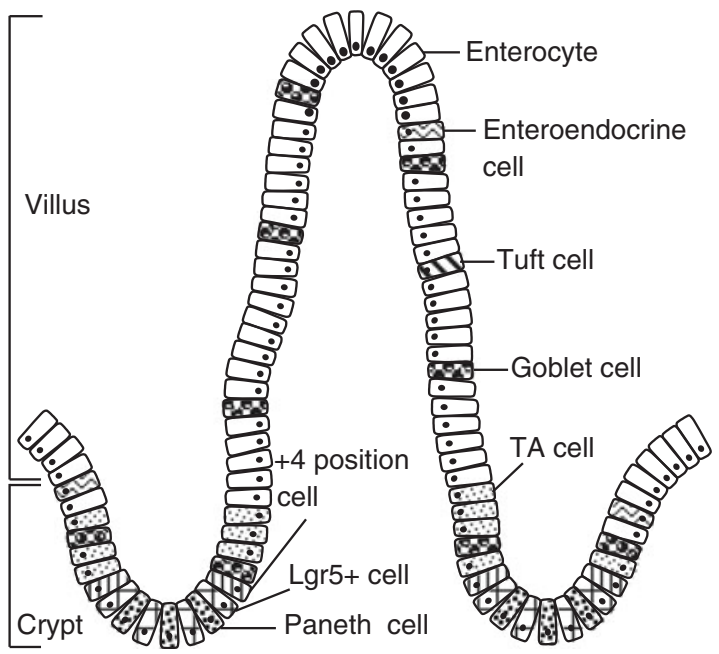

Figure 1 Porcine intestinal epithelium structure. Intestinal stem cells (ISCs) locates at the base of the crypt, which can be identified by marker gene Lgr5 (leucine-rich-repeat-containing G-protein-coupled receptor 5). The +4 position cell resides at the fourth position from the base of the crypt, above the Paneth cell. Paneth cells are located between Lgr5-positive $\left(\operatorname{Lgr}^{+}\right)$cells. The daughter cells of ISCs migrate up the crypt-villus axis and pass through a progenitor stage, forming transient amplifying (TA) cells, before becoming differentiated cells (enterocyte, enteroendocrine cell, tuft cell, goblet cell).

(Yin et al., 2014). Glutathione in reduced form (GSH) is an antioxidant that can directly react with reactive-oxygen species, generating oxidized glutathione (GSSG) through glutathione peroxidase. In turn, GGSG can be catalyzed into GSH by glutathione reductase. Since the reduced and oxidized forms of glutathione are simultaneously present in cells, the ratio of GSSG : GSH is an indicator of oxidative stress, that not only determines cellular redox status but also controls intracellular concentrations of both oxygen-reactive and nitrogen-reactive species (Cruzat et al., 2018). In 28-dayold pigs, weaning reduced feed intake $(-36 \%)$, BW gain $(-47 \%)$ and small intestine weight $(-26 \%)$ over the 7 -day post-weaning. Meanwhile, weaning increased the expression of genes $(+52 \%$ to $+346 \%)$ related to oxidative stress (i.e. glutathione peroxidase-1 and -4) and the GSSG : GSH ratio $(+59 \%)$ when compared with piglets maintained on the sow (Wang et al., 2008). Moreover, pigs weaned at 21 days of age grew more slowly $(-89 \%)$, had shorter villus height $(-42 \%)$ than piglets maintained on the sow (Wang et al., 2014). In addition, weaning can result in increased intestinal epithelial permeability by decreasing the relative expression of tight junctions including occludin and claudins (Wang et al., 2014). These affect the absorption and transportation of nutrients, disrupt barrier functions and may cause additional intestinal inflammatory responses (Wang et al., 2014).

\section{Glutamine may affect intestinal function of weaning pigs}

Glutamine is considered the most abundant free amino acid in the body, because plasma and tissue glutamine concentrations are 10 to 100 fold higher than any other amino acids (Cruzat et al., 2018). Weaning affects intestinal amino acid metabolism (Sève et al., 1986) with many of these changes related to glutamine metabolism. For example, in enterocytes of weaned pigs, L-glutamine is oxidized approximately eight times less slowly than in enterocytes isolated from suckling pigs (Darcy-Vrillon et al., 1994). Weaning also results in reduced glutamine concentrations in the jejunal lumen fluid $(-70 \%)$, jejunal tissue $(-38 \%)$, and plasma $(-30 \%)$ (Wang et al., 2008). However, the gut preferentially extracts glutamine as a source of energy within intestinal epithelial cells. For example, approximately $67 \%$ of dietary glutamine is catabolized by the small intestine (i.e. enterocytes, lymphocytes and bacteria) in swine, with the remaining glutamine entering the portal circulation ( $\mathrm{Wu}$ et al., 2011). Furthermore, glutamine is the only amino acid in arterial blood that is taken up by the small intestine of swine. Thus, although the small intestine represents only $3 \%$ to $4 \%$ of BW, it utilizes $30 \%$ of total arterial glutamine in pigs (Wu et al., 2011). Therefore, glutamine may affect intestinal development, structure and function and growth performance of weaning piglets. Therefore, the potential of feeding higher dietary glutamine levels to weaning pigs, the underlying mechanisms and how they are influenced by glutamine deserves further research.

\section{Glutamine metabolism in the small intestine}

The gut derives glutamine from both the diet and blood. The small intestine is the principal organ for glutamine uptake and metabolism into the body. L-glutamate is the principal ingested form that exerts its physiological function in the gut (Blachier et al., 2013). Dietary glutamine is taken up from the intestinal lumen and transported across the apical membranes of the intestinal enterocyte by a variety of amino acid transport systems (i.e. $\mathrm{Na}^{+}$-dependent transport systems) to cytosol and mitochondria. Following that, L-glutamine enters the mitochondria, where it is degraded to L-glutamate and ammonia by the phosphate-dependent glutaminase, which is very abundant in enterocyte mitochondria in both villus and crypt cells (Pinkus and Windmueller, 1977). Dietary glutamate serves primarily as an important energy source for rapidly dividing porcine mucosal cells (Reeds et al., 1996 and 2000; Janeczko et al., 2007). For example, in pigs weaned at 21 days of age and administered three- to fourfold the normal level of dietary glutamate, the majority (70\% to $80 \%$ ) of absorbed glutamate was utilized by the gut, and a large proportion of this served as an oxidative fuel for the mucosa (Janeczko et al., 2007).

Second, as a protein synthesis precursor, glutamine has many metabolites, including glutamate, alanine, aspartate, $\alpha$-ketoglutarate, arginine and proline (Blachier et al., 1999). Transamination (amino acid synthesis and interconversion) appears to be the principal route by which enteral glutamate is converted to $\alpha$-ketoglutarate in enterocytes in weaning pigs (Madej et al., 1999). Glutamate is metabolized into alanine and $\alpha$-ketoglutarate in the presence of pyruvate, and into aspartate and $\alpha$-ketoglutarate in the presence of oxaloacetate. Then, $\alpha$-ketoglutarate enters the Krebs cycle 


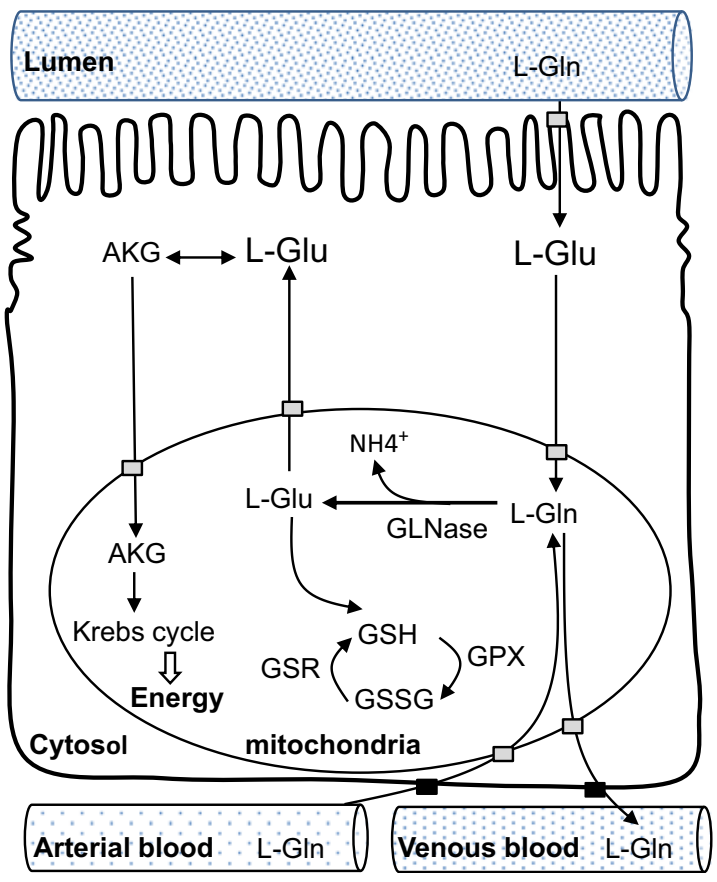

Figure 2 Schematic view of catabolic metabolism of glutamine in the small intestine, adapted from Blachier et al., (2013). AKG = alpha-ketoglutarate; $\mathrm{Gln}=$ glutamine; $\quad$ GLNase $=$ phosphate-dependent glutaminase; $\mathrm{Glu}=$ glutamate; $\mathrm{GPX}=$ glutathione peroxidase; $\mathrm{GSH}=$ reduced form of glutathione; $G S R=$ glutathione reductase; $G S S G=$ oxidized form of glutathione.

to be oxidized to produce energy. Third, less than $10 \%$ of total glutamine extracted by the pig gut is used for protein synthesis (Stoll et al., 1998). Lastly, one consequence of glutamine metabolism is to produce glutathione via glutamate. Together with L-cysteine and glycine, enteral L-glutamate is the precursor for the synthesis of glutathione, and among these three amino acids, glutamate probably represents the most important step (Cruzat et al., 2018). Most of the metabolites of glutamine are oxidized with the remaining being exported through the hepatic portal vein to the whole body (Blachier et al., 2013). Note that while cellular glutamine can be metabolized into purines, pyrimidines for the biosynthesis of nucleotides, glutamate cannot replace this function. Conversely, glutamate and ammonia can be synthesized into glutamine by glutamine synthetase in the skeletal muscles or liver, but this reaction consumes energy (Cruzat et al., 2018). A schematic view of catabolic metabolism of glutamine in small intestine is shown in Figure 2.

\section{Glutamine regulates intestinal function of weaning pigs}

Numerous studies have suggested that glutamine has positive effects on piglet growth performance, intestinal morphology, epithelial cell renewal, antioxidant capacity and barrier functions (Wu et al., 1996; Domeneghini et al., 2006; Wang et al., 2008 and 2014; Molino et al., 2012; Zhu et al., 2015; Jiang et al., 2017).
Glutamine improves piglet health, growth and intestinal morphology

Rhoads et al. (1991) reported that glutamine is an effective oral rehydration solution for rotavirus-induced diarrhea. Compared to normal newborn piglets, the rotavirus-induced diarrhea piglets had decreased villus height $(-65 \%)$, thinner mucosa and increased thymidine kinase activity $(+700 \%)$ (Rhoads et al., 1991). Meanwhile, the activities of lactase specific $(-20 \%)$, alkaline phosphatase $(-38 \%)$ and $\mathrm{Na}^{+} / \mathrm{K}^{+}$ATPase $(-50 \%)$ were all reduced in response to rotavirusinduced diarrhea (Rhoads et al., 1991). Moreover, L-glutamine (30 mmol/l) was found to stimulate in vitro absorption of sodium and chloride in rotavirus-infected pigs, returning the rates those observed in uninfected tissue (Rhoads et al., 1991). However, that study did not further investigate whether glutamine affects the villus height and mucosal enzyme activities of infected pigs. In another study, piglets that were weaned at 21 days and supplemented with $0.5 \%, 1 \%, 2 \%$ and $4 \%$ monosodium glutamate for 21 days exhibited a dose-dependent reduction in the incidence of diarrhea in the first week after weaning (Rezaei et al., 2013). These data indicate that glutamine or glutamate can alleviate diarrhea. Other studies demonstrate that glutamine or glutamate serves as conditionally essential amino acids, and that supplementation with either can improve weaning piglet growth performance (Wu et al., 1996; Molino et al., 2012) and intestinal epithelium structure and function (Rezaei et al., 2013; Wang et al., 2014). The reported effects of glutamine on the performance, gastrointestinal structure and function of weaned pigs are shown in Table 2 . The evidence from these published studies using pure glutamine or a mixture of glutamine and glutamate shows beneficial effects between $0.5 \%$ and $2.0 \%$ (by weight) throughout the post-weaning period, particularly during the first 2 weeks after weaning ( $\mathrm{Wu}$ et al., 1996; Domeneghini et al., 2006; Hsu et al., 2010; Molino et al., 2012; Teixeira et al., 2014; Wang et al., 2014). However, some studies also showed that there were no improvements on growth performance of post-weaning piglets supplemented with glutamine or glutamate. For example, a corn soybean meal diet supplemented with $1.0 \%$ glutamine did not improve villus height in piglets weaned at 18 days of age, but did improve feed efficiency (Kitt et al., 2002). Di et al. (2003) reported that when pigs weaned at 21 days of age were fed with $0.5 \%$ glutamine for 28 days, they showed a significant positive effect on ileal morphology and mucosal cell proliferation (lymphatic follicles and epithelial cells, macrophages and intra-epithelial lymphocytes). However, there were no significant differences among the groups in growth performances (Di et al., 2003). In spite of the positive effects of glutamine on small intestinal villus morphology and plasma net xylose absorptive concentration in 28-day-old weaning pigs, $1 \%$ or $2 \%$ glutamine supplementation in a corn-soybean meal diet did not significantly affect growth performance during the 21-day treatment period (Hsu et al., 2010). Similar results were obtained by (Amorim et al., 2018) who also did not observe any significant effects of $1 \%$ glutamine or $1 \%$ glutamate on growth performance variables in pigs fed a rice grit and 
Table 2 The reported effects of glutamine on the performance, gastrointestinal structure and function of weaned pigs

\begin{tabular}{ll}
\hline \hline Weaning age & Experiment treatment \\
\hline $\begin{array}{l}\text { Suckling and } \\
\text { piglets } \\
\text { weaned at } 28 \\
\text { days old }\end{array}$ & $\begin{array}{c}\text { Basal diet supplemented with } 0.5 \% \text { monosodium } \\
\text { glutamate to creep feed for suckling pigs on day } \\
\text { after farrowing until weaning, and to weaning pigs } \\
\text { during first 13 days after weaning, respectively }\end{array}$ \\
& $\begin{array}{c}\text { Corn and soybean meal basal diet supplemented with } \\
\text { dather } 0,0.2 \%, 0.6 \% \text { or } 1.0 \% \text { free glutamine, for }\end{array}$ \\
& 14 days
\end{tabular}

12.5 days old Six treatments including corn, whey, oat basal diet (control), basal diet supplemented either arginine, glutamate, citrulline, ornithine or polyamines, at levels of $0.93 \%, 6.51 \%, 0.94 \%, 0.90 \%$ and $0.39 \%$, respectively, for 12 days

21 days old Corn soybean meal, barley meal basal diet supplemented with $0,0.5 \%$ glutamine for 28 days

28 days old

Corn soybean meal basal diet supplemented with either $0,1 \%$ or $2 \%$ glutamine for 21 days

21 days old

21 days old

Phase 1: 21 to 35 days, adding $0.8 \%$ mixer of glutamine and glutamate in diets with no lactose or with $4.0 \%$ or $8.0 \%$ lactose inclusion; phase 2 : 36 to 49 days, all animals received the same diet but with no lactose inclusion in corn and soybean meal basal diet

Corn soybean meal basal diet supplemented with 0 $0.5 \%, 1 \%, 2 \%$ and $4 \%$ monosodium glutamate for 21 days

21 days old Corn soybean meal basal diet supplemented with 0 $1 \%$ L-glutamine compared with piglets reared by sows, for 7 days

21 days old
Response to supplementation

Increased suckling pigs feed intake by $36 \%$, but no increase in weaning weight; increased weaning pigs daily food intake by $10 \%$ and daily BW gain by $7 \%$ during first 13 days after weaning

$1 \%$ glutamine improved jejunal villus height by $33 \%$ during the first week, increased gain: feed ratio by $25 \%$, increased plasma concentrations of glutamine by $24 \%$, glutamate by $74 \%$ and alanine by $35 \%$ during the second week

Pigs receiving $6.51 \%$ glutamate significantly increased duodenum villus height, when compared with control group or polyamine group. That indicating that glutamate prevented weaning-induced intestinal villus atrophy

Increased ileal villus height by $23.5 \%$ and crypt depth by $51.4 \%$ and decreased the ratio of villus height to crypt depth by $18.3 \%$, increased the number of mitotic mucosal cells by $4.4 \%$ and decreased apoptosis of enterocytes by $5.6 \%$ and lymphatic follicles by $25.4 \%$

Glutamine groups tended higher villus height than the control group in duodenum and jejunum. $2 \%$ glutamine supplementation increased plasma net xylose absorptive concentration by $10 \%$ and $21 \%$, respectively, in days 7 to 14 after weaning

$0.8 \%$ mixer of glutamine and glutamate improved $7 \%$ BW and $10 \%$ weight gain of pigs from 21 to 49 days old and increased the villus height in duodenum by $7.7 \%$, jejunum by $10.4 \%$ and ileum by $6.6 \%$ at 49 days of age

$4 \%$ monosodium glutamate increased BW $7.8 \%$, daily gain $31.6 \%$, jejunal villus height $31.9 \%$, crypt depth $8.1 \%$, reduced diarrhea incidence $78.6 \%$ and enhanced jejunal concentrations of adenosine triphosphate $54.8 \%$, deoxyribonucleic acid $35.0 \%$, ribonucleic acid $30.7 \%$, during the first week after weaning

Weaned pigs in control group grew (over 7 days) 89\% Wang et al. slower than sow-reared piglets. Those offered a diet with $1 \%$ glutamine grew $68 \%$ slower than sowreared piglets. Similar effects of glutamine on villus height were also observed in the jejunum tissues. Glutamine had the adverse effect of weaning on growth, villus height but not totally overcoming the stress of weaning on intestinal structure and function

$1.0 \%$ mixtures improved weight gain by $68.3 \%$, feed intake by $29.8 \%$, decreased feed conversion ratio by $29.7 \%$ and diarrhea score by $12.1 \%$ compared with porcine plasma control group during the first week after weaning. 1.0\% mixtures also reduced duodenal crypt depth $17.3 \%$ and ileum digestive content pH $2.2 \%$, increased villus : crypt ratio $36.8 \%$ at age 28 days, when compared to the porcine plasma diet
References

Gatel and

Guion

(1990)

Wu et al.

Ewtushik

et al.

(2000)

Domeneghini

et al.

(2006)

Hsu et al.

(2010)

Molino et al.

Rezaei et al.

Teixeira et al. between glutamine and glutamate was unknown); five treatments: basal diet supplementation with 0 $0.5 \%, 1.0 \%, 1.5 \%$ mixtures and positive control (basal diet $+4 \%$ porcine plasma) for 21 days in corn and soybean meal-based diet 
soybean-based diet. Different diets, weaning age, experimental duration or dosage of glutamine may contribute to differing growth performance responses to glutamine or glutamate supplementation (Kitt et al., 2002; Di et al., 2003; Amorim et al., 2018).

\section{Glutamine and cellular redox state}

Glutamine metabolism can provide glutamate required for cellular glutathione synthesis. Glutathione can directly react with ROS and thereby protect cellular macromolecules such as DNA, proteins and lipids against oxidative stress (Cruzat et al., 2018). Glutamine has been shown to preserve total glutathione levels after injury/ischemia to gut (Harward et al., 1994). In response to cobalt chloride $(375 \mu \mathrm{mol} / \mathrm{kg}$ BW)-generated oxidative stress, rats showed increased liver thiobarbituric acid reactive substances content $(+50 \%)$ and decreased GSH (-40\%) activity compared to control values (Gonzales et al., 2005). The level of antioxidant enzymes catalase, glutathione peroxidase and superoxide dismutase in liver also was decreased by about $30 \%$ after cobalt injection, while that of hepatic heme oxygenase-1 induction was increased over 14-fold after cobalt treatment. However, these dramatic effects of cobalt on enzyme activities, including thiobarbituric acid reactive substances and GSH, were not observed in rats that had been pretreated with dietary glutamine (300 mg/kg BW). Also, the marked increase in the liver heme oxygenase-1 (protein expression and activity) due to cobalt challenge was partially prevented by glutamine administration (Gonzales et al., 2005). These data indicate that glutamine supplementation prevents glutathione depletion in the liver of rats, thus exerting protective effects during oxidant stress. Similarly, in pigs weaned at 21 days age and supplemented with $4 \%$ monosodium glutamate, there was an increase in jejunal GSH $(+56 \%)$ and a decrease in GSSG $(-24 \%)$ and the ratio of GSH : GSSG $(-54 \%)$ during the first week after weaning (Rezaei et al., 2013). Dietary glutamate also alleviated intestinal oxidative stress in weaning pigs, when animals were challenged by the potent oxidants diquat and hydrogen peroxide (Yin et al., 2015b; Duan et al., 2016). Diquat is an herbicide that generates superoxide anions through redox cycling and has been used to induce oxidative stress in vivo (Lv et al., 2012). A diquat injection to piglets significantly decreased final BW $(-22 \%)$, average daily weight gain $(-125 \%)$ and feed intake $(-57 \%)$ (Yin et al., 2015b). Meanwhile, supplementation with $2 \%$ glutamate after diquat treatment partially restored final $\mathrm{BW}$ to $91.2 \%$, average daily feed intake to about $50 \%$, and average daily weight gain to $19 \%$ of control levels (Yin et al., 2015b). Diquat disrupted antioxidant enzymes balances, such as decreased serum nitric oxide about onefold, elevated serum malondialdehyde by about $80 \%$. Diquat also decreased serum concentrations of threonine $(-42 \%)$, serine $(29 \%)$, glycine $(26 \%)$ and glutamate (-5.5\%) (Yin et al., 2015b). Pigs treated with $2 \%$ glutamate increased nitric oxide and reduced malondialdehyde. Meanwhile, 2\% supplemental glutamate significantly enhanced serum glutamate level by $21 \%$ and associated amino acid transporters expression.
Glutamate supplementation increased serum threonine, serine and glycine concentrations, although they did not return completely to control values (Yin et al., 2015b).

\section{Glutamine and intestinal epithelial cells renewal}

Glutamine and intestinal epithelial cell proliferation and differentiation. Glutamine has long been recognized as having an important role in cell survival and proliferation (Ehrensvard et al., 1949). Glutamine promotes intestinal cell proliferation in rats (Swaid et al., 2013), piglets (Wang et al., 2008) and various cell lines (Chen et al., 2018b). A recent study with weaning mice by Chen et al. (2018a) found that $10 \mathrm{mg} / \mathrm{ml}$ glutamine promotes Lgr5 (leucine-rich-repeat-containing G-protein-coupled receptor 5, also known as Gpr49), a stem cell marker, expression in intestinal cells. Another study found that Lgr5 stem cell growth is closely associated with epidermal growth factor (EGF), Wnt3 and the Notchligand. These are all essential signals required for stem cell proliferation and differentiation (Sato et al., 2011). Further work is necessary to determine if and how glutamine changes these signal pathways to promote Lgr5 stem cell development. Some studies indicate that supplemental glutamine had no effect on lysozyme and angiogenin expressions that are Paneth cell markers (Liu et al., 2017; Chen et al., 2018a). For example, glutamine at a dosage of $2 \%$ did not promote mRNA expression in other Paneth cell-associated factors such as $\alpha$-defensins (cryptdin-1, -4 and -5), lysozyme $C$ and angiogenin 4 in the ileum of mouse models (Liu et al., 2017). Glutamine at a dosage of $10 \mathrm{mg} / \mathrm{ml}$ fed to weaning mice did not affect the number of absorptive enterocytes, Paneth cells, goblet cells, enteroendocrine cells or the gene expression of these cell markers (Chen et al., 2018a). However, the in vitro administration of $10 \mathrm{mmol}$ glutamine increased chromogranin A and mucin 2 expression. These are differentiation markers for enteroendocrine and goblet cells, respectively (Chen et al., 2018b). This apparent discrepancy may result from differences between in vivo and in vitro studies. Whether glutamine affects weaning piglet intestinal cell differentiation warrants further investigation.

Glutamine and intestinal cell apoptosis. Various factors, such as glutamine deprivation (Papaconstantinou et al., 1998), toxins (Wu et al., 2014) and inflammation (Crespo et al., 2012), have been reported to induce intestinal cell apoptosis. Glutamine administered rectally to rat with colitis reduced activating transcription factor 6 (ATF6), activating transcription factor 4 (ATF4) and spliced X-box-binding protein-1 (XBP-1) mRNA levels. These are transmembrane proteins in the endoplasmic reticulum that have pro-endoplasmic reticulum stress effects. Glutamine $(25 \mathrm{mg} / \mathrm{dl})$ administration inhibited the pro-apoptotic protein expression of phospho-p53 and cytochrome $c$, and decreased activities of apoptotic markers including caspase-9, caspase-8 and caspase-3 (Crespo et al., 2012). Double immunofluorescence staining showed co-localization of C/enhancer binding protein homologous protein (CHOP) and cleaved caspase-3 in colon sections (Crespo et al., 2012). When $2 \%$ glutamate was given to piglets weaned at 
28 days of age, plasma superoxide dismutase and glutathione peroxidase activities were increased after being challenged with deoxynivalenol for 30 days (Wu et al., 2014). Significantly increasing proliferating indexes and markedly decreasing caspase- 3 activities in the jejunum and the ileum were observed in toxin-challenged piglets treated with $2 \%$ glutamate (Wu et al., 2014). These results indicate that glutamine is beneficial to alleviate intestinal cell apoptosis.

Glutamine and intestinal cell endoplasmic reticulum stress. Glutamine $(0.45 \mathrm{~g} / \mathrm{l})$ lessened endoplasmic reticulum stress in intestinal porcine epithelial cell line J2 (IPEC-J2) treated with tunicamycin, an endoplasmic reticulum stress inducer (Jiang et al., 2017). First, IPEC-J2 was exposed to tunicamycin and spontaneous endoplasmic reticulum stress was observed. It was exhibited by upregulation of the glucose-regulated protein 78 (GRP78). Second, prolonged tunicamycin treatment induced apoptosis mediated by CHOP, and this was accompanied by GRP78 downregulation (Jiang et al., 2017). Third, stressed cells that were treated with $0.45 \mathrm{~g} / \mathrm{l}$ L-glutamine maintained a high level of GRP78 while CHOP-mediated apoptosis was alleviated. The inositol requiring enzyme1 $\alpha$ (IRE1 $\alpha$ )-XBP1 axis was also activated and cell proliferation promoted. Finally, a specific inhibitor of the IRE1 $\alpha$-XBP1 axis reversed the protective effect of L-glutamine by blocking the expression of IRE1 $\alpha / X B P 1 s$ (Jiang et al., 2017). These findings indicate that L-glutamine facilitates intestinal health partly through modulating endoplasmic reticulum stress and CHOP-mediated apoptosis. These data give some insight into the mechanism by which glutamine repairs intestinal damage in weaned piglets.

Glutamine and intestinal cell autophagy. Glutamine affects autophagy and coordinates cell growth and proliferation in various cells, including cancer cells (Nicklin et al., 2009) and porcine intestinal cells (Zhu et al., 2015). Glutamine deprivation in porcine intestinal epithelial cells (IPEC-1) increased the autophagy marker light chain 3B-II in a time-dependent manner and decreased cell growth number in a time-dependent manner (Zhu et al., 2015). Glutamine supplementation at $5 \mathrm{mmol} / \mathrm{l}$ increased cell growth and cellular protein content and repressed IPEC-1 cell light chain 3B-II protein abundance (Zhu et al., 2015). Glutamine deprivation reduced mammalian target of rapamycin (mTOR) activation and its downstream protein p70S6 kinase. It reduced mitogen-activated protein kinase (MAPK) phosphorylation and its downstream extracellular signal-related kinase (ERK) 1/2 protein (Zhu et al., 2015) indicating that glutamine may suppresses cell autophagy by activating $\mathrm{mTOR}$ and MAPK signaling pathways. Nuclear factor kappa B (NF- $\kappa$ B) and nuclear factor erythroid 2-related factor 2 (Nrf2) are both known to act as transcription factors in stress adaptation and tissue protection (Ringseis et al., 2016). Weaning piglets exposed to hydrogen peroxide were found to form cell autophagosome in the jejunum (Yin et al., 2015a). Nuclear factor kappa B was triggered along with Nrf2, and Keap1, a Kelch-like ECH-associated 23 protein 1, signaling pathway (Yin et al., 2015a). When 6-week-old pigs were fed a diet with oxidized fat (rapeseed oil that had been heated to $175^{\circ} \mathrm{C}$ for $72 \mathrm{~h}$ ) for 29 days, mRNA concentrations in Nrf2 target genes, including $\mathrm{NAD}(\mathrm{P}) \mathrm{H}$ dehydrogenase, quinone 1 , peroxiredoxin 6 , glutathione peroxidase 2 and thioredoxin reductase 1 in the duodenal mucosa, were upregulated (Ringseis et al., 2016). Protein concentrations of active or cleaved caspase- 3 in the duodenal mucosa increased approximately 30\% (Ringseis et al., 2016). The association between glutamine regulation of piglet small intestinal autophagy and Nrf2/Keap1 signal pathway remains unknown.

\section{Glutamine and intestinal paracellular permeability}

The intestinal epithelium provides several extrinsic and intrinsic defense mechanisms. One is the permeability barrier that is regulated predominantly by tight junctions (Turner, 2009). The beneficial effect of glutamine on intestinal paracellular permeability has been well described in humans, animals (Wang et al., 2014) and various cell lines (Li and Neu, 2009; Wang et al., 2016a). For example, glutamine depletion results in porcine villus atrophy (Wu et al., 1996). Intestinal permeability was increased by $40 \%$ by weaning (Wang et al., 2014), and this was accompanied by villus atrophy and reduced jejunal protein expression, including occludin, claudin-1, zonula occludens-2 (ZO-2) and ZO-3. Dietary $1 \%$ L-glutamine supplementation attenuated the weaning stress-induced mucosal barrier dysfunctions although it did not return growth performance, villus morphology, tight junction protein expression (occludin, claudin-1, zonula occludens-2) to levels exhibited by sow-reared piglets (Wang et al., 2014). The underlying mechanism for glutamine's regulatory effect on paracellular permeability may involve multiple signal pathways. Glutamine at $4.0 \mathrm{mmol} / \mathrm{l}$ may regulate claudin-expression via the PI3K/AKT pathway in Caco-2 cells (Li and Neu, 2009). Glutamine at 2.0 $\mathrm{mmol} / \mathrm{l}$ was found to regulate claudin-1, claudin- 4 and ZO-1 distributions through activated phosphatidylinositol 3-kinase (PI3K)/protein kinase B (AKT) but via CaMKK2AMPK, calmodulin-dependent kinase 2 to AMP-activated protein kinase, in IPEC-1 cells (Wang et al., 2016a). One recent study observed that $2.0 \mathrm{mmol} / \mathrm{l}$ glutamine prevented EGF receptor degradation and activated EGF receptor tyrosine kinase in intestinal epithelial-6 cells. Glutaminemediated EGF receptor signaling activated ERK1/2 and inactivated p38MAPK in the heat-stressed cells (Niederlechner et al., 2013). These results suggest that glutamine is an important metabolic checkpoint and may have distinct regulating pathways in different cells. The type of glutamine being added may influence outcomes. When Caco- 2 cells are treated with acetaldehyde $(600 \mu \mathrm{mol})$, there is a time-dependent decrease in transepithelial electrical resistance and an increase in inulin permeability. However, supplementation with L-glutamine $(2 \mathrm{mmol})$ effectively prevented the acetaldehyde-induced changes in transepithelial electrical resistance and inulin permeability. Interestingly, D-Glutamine $(2 \mathrm{mmol})$ or a glutaminase inhibitor by themselves did not influence transepithelial electrical resistance or inulin flux in control or acetaldehyde-treated Caco-2 cell 
monolayers (Seth et al., 2004). Glutaminase is responsible for catalysing glutamine into glutamate and ammonia suggesting that L-glutamine rather than D-glutamine or glutamate protects the barrier function in Caco-2 cell monolayers from acetaldehyde-induced injury.

\section{Conclusions}

Despite glutamine not being considered essential in the traditional sense, there is growing evidence that glutamine supplementation alleviates the negative effects induced by weaning. A healthy gut is extremely important for animal growth and development. Glutamine is a functional amino acid involved in many metabolic processes that are especially important for small intestinal health and development. Our review addressed the mechanisms involved and the physiological significance for dietary glutamine in small intestine of weaning pigs. This nutrient is necessary to support maximum growth by reducing weaning piglet intestinal oxidative stress, preventing intestinal epithelium damage and improving normal barrier function.

\section{Acknowledgements}

This work was supported by the National Natural Science Foundation of China (No. 31330075, No. 31402089, No. 31640078, No. 31872991), the Key Programs of Frontier Scientific Research of the Chinese Academy of Sciences (QYZDY-SSW-SMC008), the Key Project of Hunan Provincial Education Department (16A096) and the Innovation Platform and Talents Program of Hunan Provincial Science and Technology Department (2018RS3105) and Central Public-interest Scientific Institution Basal Research Fund for Chinese Academy of Tropical Agricultural Sciences (No. 1630032019022).

(1) H. S. Yang 0000-0003-1164-5771

\section{Declaration of interest}

None.

\section{Ethics statement}

All ethical standards have been met.

\section{Software and data repository resources}

None.

\section{References}

Amorim AB, Saleh MAD, Miassi GDM and Berto DA 2018. Dietary supplementation with glutamine or glutamic acid for weanling piglets. Pesquisa Agropecuária Brasileira 53, 229-237.

Blachier F, Guihot-Joubrel G, Vaugelade P, Le Boucher J, Bernard F, Duée PH and Cynober L 1999. Portal hyperglutamatemia after dietary supplementation with monosodium glutamate in pigs. Digestion 60, 349-357.

Blachier F, Wu G, Yin Y, Hou Y and Andriamihaja M 2013. Developmental amino acid metabolism in the pig small and large intestine epithelial cells. In Nutritional and physiological functions of amino acids in pigs (ed. F Blachier, G Wu and Y Yin), pp. 59-74. Springer, Vienna.
Boudry G, Péron V, Huërou-Luron IL, Lallès JP and Sève B 2004. Weaning induces both transient and long-lasting modifications of absorptive, secretory, and barrier properties of piglet intestine. Journal of Nutrition 134, 2256-2262.

Chen S, Xia Y, Zhu G, Yan J, Tan C, Deng B, Deng J, Yin Y and Ren W 2018a. Glutamine supplementation improves intestinal cell proliferation and stem cell differentiation in weanling mice. Food \& Nutrition Research 62, 1439.

Chen Y, Tseng SH, Yao CL, Li C and Tsai YH 2018b. Distinct effects of growth hormone and glutamine on activation of intestinal stem cells. Journal of Parenteral \& Enteral Nutrition 42, 642-651.

Crespo I, San-Miguel B, Prause C, Marroni N, Cuevas MJ, González-Gallego J and Tuñón MJ 2012. Glutamine treatment attenuates endoplasmic reticulum stress and apoptosis in TNBS-induced colitis. PLoS ONE 7, e50407.

Cruzat V, Macedo Rogero M, Noel Keane K, Curi R and Newsholme P 2018. Glutamine: metabolism and immune function, supplementation and clinical translation. Nutrients 10, 1564.

Darcy-Vrillon B, Posho L, Morel MT, Bernard F, Blachier F, Meslin JC and Duée PH 1994. Glucose, galactose, and glutamine metabolism in pig isolated enterocytes during development. Pediatric Research 36, 175-181.

Di Giancamillo A, Domeneghini C, Paratte R, Dell'Orto V and Bontempo V 2003. Oral feeding with L-glutamine and nucleotides: impact on some GALT (gut associated lymphoid tissue) parameters and cell proliferation/death rates in weaning piglets. Italian Journal of Animal Science 2 (suppl. 1), 364-366.

Domeneghini C, Giancamillo AD, Bosi G and Arrighi S 2006. Can nutraceuticals affect the structure of intestinal mucosa? Qualitative and quantitative microanatomy in L-glutamine diet-supplemented weaning piglets. Veterinary Research Communications 30, 331-342.

Duan J, Yin J, Ren W, Liu T, Cui Z, Huang X, Wu L, Kim SW, Liu G and Wu X 2016. Dietary supplementation with L-glutamate and L-aspartate alleviates oxidative stress in weaned piglets challenged with hydrogen peroxide. Amino Acids 48, 53-64.

Ehrensvard G, Fischer A and Stjernholm R 1949. Protein metabolism of tissue cells in vitro; the chemical nature of some obligate factors of tissue cell nutrition. Acta Physiologica 18, 218-230.

Ewtushik AL, Bertolo RFP and Ball RO 2000. Intestinal development of earlyweaned piglets receiving diets supplemented with selected amino acids or polyamines. Canadian Journal of Animal Science 80, 653-662.

Gatel F and Guion P 1990. Effect of monosodium L glutamate on diet palatability and piglet performance during the suckling and weaning periods. Animal Production 50, 365-372.

Gonzales S, Polizio AH, Erario MA and Tomaro ML 2005. Glutamine is highly effective in preventing in vivo cobalt-induced oxidative stress in rat liver. World Journal of Gastroenterology 11, 3533-3538.

Harward TRS, Coe D, Souba WW, Klingman N and Seeger JM 1994. Glutamine preserves gut glutathione levels during intestinal ischemia/reperfusion. Journal of Surgical Research 56, 351-355.

Hsu CB, Huang HJ, Wang CH, Yen HT and Yu B 2010. The effect of glutamine supplement on small intestinal morphology and xylose absorptive ability of weaned piglets. African Journal of Biotechnology 9, 7003-7008.

Janeczko MJ, Stoll B, Chang X, Guan X and Burrin DG 2007. Extensive gut metabolism limits the intestinal absorption of excessive supplemental dietary glutamate loads in infant pigs. Journal of Nutrition 137, 2384-2390.

Jiang Q, Chen J, Liu S, Liu G, Yao K and Yin Y 2017. L-glutamine attenuates apoptosis induced by endoplasmic reticulum stress by activating the IRE1 $\alpha$-XBP1 axis in IPEC-J2: a novel mechanism of L-glutamine in promoting intestinal health. International Journal of Molecular Sciences 18, 2617.

Kitt SJ, Miller PS, Lewis A and Fischer RL 2002. Effects of glutamine on growth performance and small intestine villus height in weanling pigs. Nebraska Swine Reports 82, 29-32.

Li N and Neu J 2009. Glutamine deprivation alters intestinal tight junctions via a PI3-K/Akt mediated pathway in Caco-2 cells. Journal of Nutrition 139, 710-714. Liu G, Ren W, Fang J, Hu CA, Guan G, Al-Dhabi NA, Yin J, Duraipandiyan V, Chen $S$ and Peng $Y$ 2017. L-glutamine and l-arginine protect against enterotoxigenic Escherichia coli infection via intestinal innate immunity in mice. Amino Acids 49, 1-10.

Lv M, Yu B, Mao XB, Zheng P, He J and Chen DW 2012. Responses of growth performance and tryptophan metabolism to oxidative stress induced by diquat in weaned pigs. Animal 6, 928-934. 
Madej M, Lundh T and Lindberg JE 1999. Activities of enzymes involved in glutamine metabolism in connection with energy production in the gastrointestinal tract epithelium of newborn, suckling and weaned piglets. Biology of the Neonate 75, 250-258.

Mccracken BA, Spurlock ME, Roos MA, Zuckermann FA and Gaskins HR 1999 Weaning anorexia may contribute to local inflammation in the piglet small intestine. Journal of Nutrition 129, 613-619.

Moeser AJ, Klok CV, Ryan KA, Wooten JG, Little D, Cook VL and Blikslager AT 2007. Stress signaling pathways activated by weaning mediate intestinal dysfunction in the pig. American Journal of Physiology-Gastrointestinal and Liver Physiology 292, G173-G181.

Molino JP, Donzele JL, de Oliveira RFM, Saraiva A, Haese D, Fortes El and de Souza MF 2012. L-glutamine and L-glutamate in diets with different lactose levels for piglets weaned at 21 days of age. Revista Brasileira de Zootecnia 41, 98-105.

Montagne L, Boudry G, Favier C, Huërouluron IL, Lallès JP and Sève B 2007. Main intestinal markers associated with the changes in gut architecture and function in piglets after weaning. British Journal of Nutrition 97, 45-57.

Nicklin P, Bergman P, Zhang B, Triantafellow E, Wang H, Nyfeler B, Yang H, Hild $M$, Kung $C$ and Wilson $C$ 2009. Bidirectional transport of amino acids regulates mTOR and autophagy. Cell 136, 521-534.

Niederlechner S, Baird C, Petrie B, Wischmeyer E and Wischmeyer PE 2013. Epidermal growth factor receptor expression and signaling are essential in glutamine's cytoprotective mechanism in heat-stressed intestinal epithelial-6 cells. American Journal of Physiology-Gastrointestinal and Liver Physiology 304, G543-G552.

Papaconstantinou HT, Hwang KO, Rajaraman S, Hellmich MR, Townsend CM Jr and Ko TC 1998. Glutamine deprivation induces apoptosis in intestinal epithelia cells. Surgery 124, 152-160.

Pinkus LM and Windmueller HG 1977. Phosphate-dependent glutaminase of small intestine: localization and role in intestinal glutamine metabolism. Archives of Biochemistry and Biophysics 182, 506-517.

Pluske JR, Hampson DJ and Williams IH 1997. Factors influencing the structure and function of the small intestine in the weaned pig: a review. Livestock Production Science 51, 215-236.

Reeds PJ, Burrin DG, Jahoor F, Wykes L, Henry J and Frazer EM 1996. Enteral glutamate is almost completely metabolized in first pass by the gastrointestinal tract of infant pigs. American Journal of Physiology 270, E413-E418.

Reeds PJ, Burrin DG, Stoll B and Jahoor F 2000. Intestinal glutamate metabolism. Journal of Nutrition 130, 978S-982S.

Rezaei R, Knabe DA, Tekwe CD, Dahanayaka S, Eide SJ, Lovering SL, Ficken MD, Fielder SE and Wu G 2013. Dietary supplementation with monosodium glutamate is safe and improves growth performance in postweaning pigs. Amino Acids 44, 911-923.

Rhoads JM, Keku EO, Quinn J, Woosely J and Lecce JG 1991. L-glutamine stimulates jejunal sodium and chloride absorption in pig rotavirus enteritis Gastroenterology 100, 683-691.

Ringseis R, Kynast AM, Couturier A, Most E and Eder K 2016. Ingestion of frying fat leads to activation of the endoplasmic reticulum stress-induced unfolded protein response in the duodenal mucosa of pigs. Molecular Nutrition \& Food Research 60, 957-963.

Sato T, van Es JH, Snippert HJ, Stange DE, Vries RG, van den Born M, Barker N Shroyer NF, van de Wetering M and Clevers H 2011. Paneth cells constitute the niche for Lgr5 stem cells in intestinal crypts. Nature 469, 415-418.

Seth A, Basuroy S, Sheth P and Rao RK 2004. L-Glutamine ameliorates acetaldehyde-induced increase in paracellular permeability in Caco-2 cell monolayer. American Journal of Physiology-Gastrointestinal and Liver Physiology 287, G510-G517.

Sève B, Reeds PJ, Fuller MF, Cadenhead A and Hay SM 1986. Protein synthesis and retention in some tissues of the young pig as influenced by dietary protein intake after early-weaning. Possible connection to the energy metabolism. Reproduction Nutrition Development 26, 849-861.

Spreeuwenberg MA, Verdonk JM, Gaskins HR and Verstegen MW 2001. Small intestine epithelial barrier function is compromised in pigs with low feed intake at weaning. Journal of Nutrition 131, 1520-1527.
Stoll B, Henry J, Reeds PJ, Yu H, Jahoor F and Burrin DG 1998. Catabolism dominates the first-pass intestinal metabolism of dietary essential amino acids in milk protein-fed piglets. Journal of Nutrition, 128, 606-614.

Swaid F, Sukhotnik I, Matter I, Berkowitz D, Hadjittofi C, Pollak Y and Lavy A 2013. Dietary glutamine supplementation prevents mucosal injury and modulates intestinal epithelial restitution following acetic acid induced intestina injury in rats. Nutrition \& Metabolism 10, 53.

Teixeira AD 0., Nogueira ET, Kutschenko M, Rostagno HS and Lopes DC 2014. Inclusion of glutamine associated with glutamic acid in the diet of piglets weaned at 21 days of age. Revista Brasileira De Saude E Producao Animal $15,881-896$

Turner JR 2009. Intestinal mucosal barrier function in health and disease. Nature Reviews Immunology 9, 799.

Wang B, Wu Z, Ji Y, Sun K, Dai Z and Wu G 2016a. L-glutamine enhances tight junction integrity by activating CaMK Kinase 2-AMP-activated protein kinase signaling in intestinal porcine epithelial cells. Journal of Nutrition 146 501-508.

Wang H, Zhang C, Wu G, Sun Y, Wang B, He B, Dai Z and Wu Z 2014. Glutamine enhances tight junction protein expression and modulates corticotropinreleasing factor signaling in the jejunum of weanling piglets. Journal of Nutrition 145, 25-31.

Wang J, Chen L, Li P, Li X, Zhou H, Wang F, Li D, Yin Y and Wu G 2008. Gene expression is altered in piglet small intestine by weaning and dietary glutamine supplementation. Journal of Nutrition 138, 1025.

Wang J, Zeng L, Tan B, Li G, Huang B, Xiong X, Li F, Kong X, Liu G and Yin Y 2016b. Developmental changes in intercellular junctions and Kv channels in the intestine of piglets during the suckling and post-weaning periods. Journal of Animal Science and Biotechnology 7, 4.

Wijtten PJ, Van der Meulen J and Verstegen MW 2011. Intestinal barrier function and absorption in pigs after weaning: a review. British Journal of Nutrition 105, 967-981

Wu G 2014. Dietary requirements of synthesizable amino acids by animals: a paradigm shift in protein nutrition. Journal of Animal Science and Biotechnology 5, 34.

Wu G, Bazer FW, Burghardt RC, Johnson GA, Kim SW, Knabe DA, Li X, Satterfield MC, Smith SB and Spencer TE 2010. Functional amino acids in swine nutrition and production. In Dynamics in animal nutrition (ed. J Doppenberg and $P$ van der Aar), pp. 69-98. Wageningen Academic Publishers, The Netherlands.

Wu G, Bazer FW, Johnson GA, Knabe DA, Burghardt RC, Spencer TE, Li XL and Wang JJ 2011. Triennial growth symposium: important roles for Lglutamine in swine nutrition and production. Journal of Animal Science 89, 2017-2030.

Wu G, Meier SA and Knabe DA 1996. Dietary glutamine supplementation prevents jejunal atrophy in weaned pigs. Journal of Nutrition 126, 2578-2584.

Wu M, Xiao H, Ren W, Yin J, Hu J, Duan J, Liu G, Tan B, Xiong X and Oso A 0 2014. An NMR-based metabolomic approach to investigate the effects of supplementation with glutamic acid in piglets challenged with deoxynivalenol. PLOS ONE 9, e113687.

Yang $H$, Wang $X$, Xia $X$ and Yin Y 2016a. Energy metabolism in intestinal epithelial cells during maturation along the crypt-villus axis. Scientific Reports 6 , 31917

Yang $\mathrm{H}$, Xiong X, Wang X, Tan B, Li T and Yin Y 2016b. Effects of weaning on intestinal upper villus epithelial cells of piglets. PLOS ONE 11, e0150216.

Yin J, Duan J, Cui Z, Ren W, Li T and Yin Y 2015a. Hydrogen peroxide-induced oxidative stress activates NF-kB and Nrf2/Keap1 signals and triggers autophagy in piglets. Royal Society of Chemistry Advances 5, 15479-15486.

Yin J, Liu M, Ren W, Duan J, Yang G, Zhao Y, Fang R, Chen L, Li T and Yin Y $2015 \mathrm{~b}$. Effects of dietary supplementation with glutamate and aspartate on diquat-induced oxidative stress in piglets. PLoS ONE 10, e0122893.

Yin J, Wu MM, Xiao H, Ren WK, Duan JL, Yang G, Li TJ and Yin YL 2014 Development of an antioxidant system after early weaning in piglets. Journal of Animal Science 92, 612.

Zhu Y, Lin G, Dai Z, Zhou T, Li T, Yuan T, Wu Z, Wu G and Wang J 2015. L-Glutamine deprivation induces autophagy and alters the mTOR and MAPK signaling pathways in porcine intestinal epithelial cells. Amino Acids 47, 2185-2197. 